\title{
CORRESPONDENCE
}

\author{
The Editor, The Journal of the Royal Aeronautical Society.
}

\section{The Solution of Chitty and Southwell's Determinantal Equation

$$
\Delta_{n}=0
$$

Dear Sir,-I venture to suggest another solution of the equation

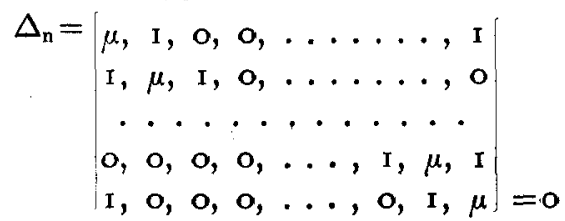

(See December issue of the Journal, I93r.)

It depends upon an artifice similar to that used by Mr. Morris (see January issue of the Journal) in his evaluation of the determinant. The artifice, however, is applied to each of the simultaneous equations which give rise to the determinant. The determinant with its evaluation is not then required. Each of the $n$ equations whose solution gives rise to the equation $\Delta_{n}=0$ is of the form $x_{r-1}+\mu x_{\mathrm{r}}+x_{\mathrm{r}+1}=0, r$ having the values $\mathrm{I}, 2,3, \ldots n$, while $x_{0} \equiv x_{\mathrm{n}}$ and $x_{n+1} \equiv x_{1}$. The indefinite solution in which all $x$ 's $=0$ and $\mu=$ arbitrary, does not necessitate $\Delta_{n}=0$, and must be excluded.

The $n$ equations each containing $\mu$ involve $n+2$ quantities, and $x_{0}$, $x_{1} \ldots x_{n_{1}}$. Hence the general formula for the value of every $x$ as a function of $\mu$ must contain two arbitrary constants in order that it may be a complete solution. Now from the identity

$$
\cos (r-\mathrm{I} \theta+c)-2 \cos \theta(\cos r \theta+c)+\cos (\overline{r+1} \theta+c)=0,
$$

we see that each of the last-mentioned $n$ simultaneous equations will be satisfied by $x_{\mathrm{r}}=A \cos (r \theta+c)$ where $\cos \theta=-\mu / 2$, and $A$ and $c$ are constants. In this every $x$ is a function of $\theta$ or $\mu$ and involves two arbitrary constants $A$ and $c$. It is therefore the general solution of the $x$ 's in terms of $\mu$.

[In order that $-2 \cos \theta$, which equals $\mu$, may cover all possible values of $\mu$ other than those between +2 and $-2, \theta$ may be given imaginary values, or we may use " $\cosh "$ instead of " $\cos "$ in the various equations. This, however, will be found unnecessary.]

We have further to satisfy $x_{n}=x_{0}$ and $x_{n+1}=x_{1}$.

These give $\cos (n \theta+c)=\cos c$ whence $n \theta=2 S \pi$ or $2 S \pi-2 c$, and $\cos \overline{(n+\mathrm{I}} \theta+c)$ $=(\cos \theta+c)$ whence $n \theta=2 S^{\prime} \pi$ or $2 S^{\prime} \pi-2 c-2 \theta$.

Therefore $\theta=S(2 \pi / n)$ and $c$ has any value or

$$
\theta=S^{\prime} \pi \text { and } c=S^{\prime \prime} \pi-n S^{\prime}(\pi / 2) \text { where } S, S^{\prime}, S^{\prime \prime}, \ldots
$$

are any integers.

The second value of $\theta$, and also that of $c$, are included in the first, unless $n S^{\prime}$ is odd. In this case we should have $x_{r}=\left(A \cos S^{\prime \prime \prime} \pi\right.$-odd $\left.\pi / 2\right)=0$, hence this solution as mentioned above must be excluded. Therefore the general solution

$$
\left.\begin{array}{l}
\text { is } \mu=-2 \cos \theta=-2 \cos S_{2 \pi} / n \\
\text { and } x_{\mathrm{r}}=A \cos \left\{r\left(S_{2 \pi / n)+C}\right\}\right.
\end{array}\right\}
$$


where $S$ is any integer and $A$ and $c$ are arbitrary constants. The general equation for $x_{\mathrm{r}}$ may be written

$$
x_{\mathbf{r}}=A \cos \{r(S 2 \pi / n)\}+B \sin \{r(S 2 \pi / n)\}
$$

which shows that the infinite number of sets of values of the $x$ 's may be resolved into the addition of two sets of values.

Giving the arbitrary constants $A$ and $c$ any particular values there will, in general, be $n$ different sets of vaiues of the $x$ 's, which are obtained by giving $S$ the values $1,2,3, \ldots n$. These must correspond to the $n$ roots of the determinantal equation, giving $\mu$. Hence these roots are the values of $-2 \cos (S 2 \pi / n)$ when $S$ is given the values $1,2,3, \ldots n$. It will be seen that except for the value -2 , the roots are duplicated.

Yours faithfully,

T. W. K. Clarke.

The Editor, 'The Journal of the Royal Alronautical Society.

Dear Sir,-With reference to Mr. J. L. Nayler's most interesting article in the March issue of the Aeronautical Journal on "The Effect of Accelerations on Human Beings," I would draw attention to the fact that there is a considerable mass of information which has been obtained by means of Sir Hiram Maxim's captive flying machines, which were erected at the Crystal Palace, Earl's Court, Southport, Brussels and elsewhere.

The experimental machine, erected in the grounds of Thurloe Park, was speeded up during its trials in 1904 until the resultant force upon the cars was 6.87 times gravity. This force was calculated from a careful record of the speed of the machine. I was in one of the cars during these trials and became unconscious at the maximum speed and fell to the bottom of the car. My companion, however, one of the wotkmen, managed to signal to the engine-house to stop, and the machine was brought slowly to a standstill. I regained consciousness when the speed had fallen so that the resultant force was about three times gravity. No ill effects were felt within a few minutes of the experience. Although my companion was able to signal after I had become unconscious, I am sure, from my questioning of him, that he was only able to act at great effort and was nearly unconscious himself. Since that time I have made every form of evolution in the air and have never experienced any discomfort from the accelerations. Hence it would appear that the accelerations of aerial acrobatics are considerably less than 6.87 times gravity.

In the Maxim Captive Flying Machine the speed is slowly increased, and therefore one is under the action of centrifugal force for a considerable time. In other words, the test is probably more exacting than in normal evolutions in the air experiencing the same maximum accelerations. From my own experience it: would appear that it is not possible for human beings to endure consciously, for more than a few minutes, centrifugal force along the line of the spine greater than from six to seven times gravity.

I should welcome further information upon the effect of accelerations on human beings, not only in the line of the spine but also transverse to the spine, and the effects not only on the heart and brain but also upon the eyes.

$$
\text { Yours faithfully, }
$$

8th March, 1932.

A. P. Thurston. 